\title{
Case - Laparoscopic transperitoneal partial nephrectomy of T3a renal cell carcinoma within a horseshoe kidney
}

\author{
Miles Mannas ${ }^{1}$; Michael Eng ${ }^{1}$; Ryan Flannigan ${ }^{2}$ \\ ${ }^{1}$ Department of Urologic Sciences, Unversity of British Columbia, Vancouver, BC, Canasa; ${ }^{2}$ Department of \\ Urology, Weill Cornell Medicine, New York, NY, United States
}

Cite as: Can Urol Assoc J 2018 Feb. 6; Epub ahead of print. http://dx.doi.org/10.5489/cuaj.4781

\section{Published online February 6, 2018}

$* * *$

\section{Introduction}

Horseshoe kidney (HSK) is a benign malformation characterized by three anatomic abnormalities: ectopia, malrotation, and vascular changes. ${ }^{1}$ Renal cell carcinoma (RCC) comprises approximately $53.8 \%$ of HSK malignancies. The incidence of RCC within HSK is predicted to equal that within the general population, approximately 5.2/100 000 individuals. $^{2-4}$

Surgical resection of these tumors has been described in the literature. Evidence is mounting that partial nephrectomy, rather than radical nephrectomy, and minimally invasive techniques for T3a RCC is safe and attains equivalent oncologic outcomes. ${ }^{5,6}$ Review of the literature reveals no case reports of laparoscopic partial nephrectomy for T3a RCC, and therefore, this is the first report of a laparoscopic partial nephrectomy of T3a RCC HSK with renal vein tumor thrombus.

\section{Case report}

A 49 year old male presented for kidney donation. Upon workup with abdominal computed tomography (CT) he was found to have a HSK in addition to a 6.9 x 5.6 x 5.9 $\mathrm{cm}$ right sided renal mass suspicious for RCC.

The patient was asymptomatic with neither flank or abdominal pain, nor gross hematuria; however, he did describe weekly night sweats for the preceding 6 months. His body mass index was $28.9 \mathrm{~kg} / \mathrm{m}^{2}$ and physical examination revealed genitalia revealed a right-sided grade 2 varicocele, and a left sided grade 3 varicocele, testes and phallus were otherwise unremarkable.

Review of the imaging led to a clinical staging of cT3aN0M0. A tumor thrombus was present within the most cephalad right renal vein extending to $1.6 \mathrm{~cm}$ from the insertion to the IVC. There were two co-dominant right renal veins. The RENAL 
nephrometry score was $10 x h$. Renal function was normal with serum creatinine of 77 $\mu \mathrm{mol} / \mathrm{L}$ for an estimated GFR of $93 \mathrm{ml} / \mathrm{min}$. Several management options were considered including partial versus radical nephrectomy and laparoscopic versus open approaches. It was decided that a laparoscopic partial nephrectomy was feasible and the procedure of choice.

Intra-operatively, the patient was positioned in left lateral decubitus. Five laparoscopic ports were placed on the right-side of the abdomen. The colon and duodenum were mobilized. The inferior vena cava was identified and dissection of the hilum and upper pole was performed. A concerted effort was made to identify and dissect the segmental branches of the hilar renal arteries and veins. An intra-operative laparoscopic ultrasound probe was used to identify the margins of the tumor and the extent of the renal vein tumor thrombus, which was also visualized grossly and limited to the segmental renal vein. No milking or manipulation of the tumor thrombus was necessary. A 35mm vascular cutting stapler was used to transect the cephalad renal vein proximal to the tumor thrombus (Figure 3A). Laparoscopic bulldogs were used to selectively clamp the segmental branches of the right renal artery. Absence of doppler flow to the tumor and mid/upper pole of the right kidney was confirmed with the laparoscopic US probe (Figure 3B). The lower pole of the right kidney remained perfused throughout the entire resection. Resection with laparoscopic metzenbaum scissors was performed with a thin parenchymal margin surrounding the tumor. The collecting system was then closed with 3-0 Vicryl SH, as were the open ended vessels including an arteriotomy of a segmental renal artery in the resection bed (Figure 3C). The bulldog clamps were released after 24 minutes with excellent hemostasis using the early unclamp/ selective clamping technique. Two parenchymal sutures using 0 -Vicryl were then secured using LaparaTy. Floseal was applied to the renal resection bed (Figure 3D). The specimen was extracted in a $15 \mathrm{~mm}$ Endocatch bag through extension of the right lower quadrant port. Estimated blood loss was $300 \mathrm{~mL}$.

Pathology of the specimen revealed a 5.6 x $4.8 \mathrm{~cm}$ pT3a clear cell renal cell carcinoma with extension into the renal sinus and renal vein tumor thrombus. Surgical margins were negative. There were no other adverse pathologic features. The patient has had routine clinical and radiologic follow-up with no evidence of recurrence after three years of follow-up. At three years post-operative, his renal function remains stable with a creatinine of $89 \mu \mathrm{mol} / \mathrm{L}$ (eGFR $86 \mathrm{ml} / \mathrm{min}$ ).

\section{Discussion}

Partial nephrectomy is the preferred surgical option when technically amenable for renal tumors. Comparative series have demonstrated that partial nephrectomies are non-inferior to radical nephrectomies with respect to oncological results and provide additional benefits to the patient. ${ }^{7}$ Evidence continues to accumulate that laparoscopic and robotic 
approaches for advanced RCC with renal vein tumor thrombus, is safe in properly selected patients. ${ }^{8}$ In some cases, partial nephrectomies for RCC's with renal vein tumor thrombus involvement have required milking of tumor thrombus or vascular control of IVC for adequate resection and repair after tumor thrombus removal from the renal vein; however, this is was not necessary in the present case since the renal vein could be stapled proximal to the tumor thrombus. ${ }^{8}$

Surgical resection of RCC in HSK has been described in the literature. ${ }^{9}$ HSK vascular anomalies are known to exist and make dissection and effective clamping challenging. However, the presence of multiple renal veins in this case allowed us to ligate one of the co-dominant renal veins without compromising venous outflow of the remaining right renal parenchyma. In other words, if this patient had a single right renal vein, then a partial nephrectomy would not have been technically feasible. . Careful preoperative planning and intra-operative correlation with pre-operative imaging was essential to the success of this unique and complex case. A concerted effort to identify and dissect segmental hilar right renal arteries was crucial for control and resection. Intraoperative ultrasound also aided in the safe and effective resection of tumor within renal parenchyma, and identification of the tumor thrombus to facilitate subsequent resection with clear margins. Finally, early unclamp/selective clamping technique allowed for complex resection and reconstruction while minimizing warm ischemia . To our knowledge, the present study describes the first laparoscopic transperitoneal partial nephrectomy of $6.9 \mathrm{~cm}$ T3a RCC with renal vein tumor thrombus. Three year follow-up has shown excellent oncologic control with no evidence of recurrent disease and stable renal function. 


\section{References}

1. Natsis K, Piagkou M, Skotsimara A, et al. Horseshoe kidney: a review of anatomy and pathology. Surg Radiol Anat 2014;36:517-26.

2. Buntley D. Malignancy Associated With Horseshoe Kidney. Urology 1976;8:1468.

3. Reed H and Robinson N. Horseshoe kidney with simultaneous occurrence of calculi, transitional cell and squamous cell carcinoma. Urology 1984;23:62-4.

4. Tkocz M and Kupajski M. Tumour in horseshoe kidney - different surgical treatment shown in five example cases. Contemp Oncol 2012;16:254-7.

5. Andrade HS, Zargar H, Akca O, et al. Is robotic partial nephrectomy safe for T3a renal cell carcinoma? Experience of a high-volume center. J Endourol 2017;31:153-7.

6. Patel P, Nayak J, Liu Z, et al. A multi-centered, propensity matched analysis comparing laparoscopic and open surgery for pT3a renal cell carcinoma. $J$ Endourol 2017.

7. Kunath F, Schmidt S, Miernik A, et al. Partial nephrectomy versus radical nephrectomy for clinical localised renal masses. Cochrane Database Syst Rev 2017.

8. Bansal RK, Tu HY, Drachenberg D, et al. Laparoscopic management of advanced renal cell carcinoma with renal vein and inferior vena cava thrombus. Urology 2014;83:812-6.

9. Reboucas RB, Monteiro RC, Souza TN, et al. Pure laparoscopic radical heminephrectomy for a large renal-cell carcinoma in a horseshoe kidney. Int Braz J Urol 2013;39:604-5. 


\section{Figures and Tables}

Fig. 1. Abdominal CT scan with IV contrast. The mass is $5.9 \mathrm{~cm}$ transverse, located in the right upper/interpolar region. The mass enhances with intravenous contrast, and arrow identifies a renal vein thrombosis in the most cephalad vein.

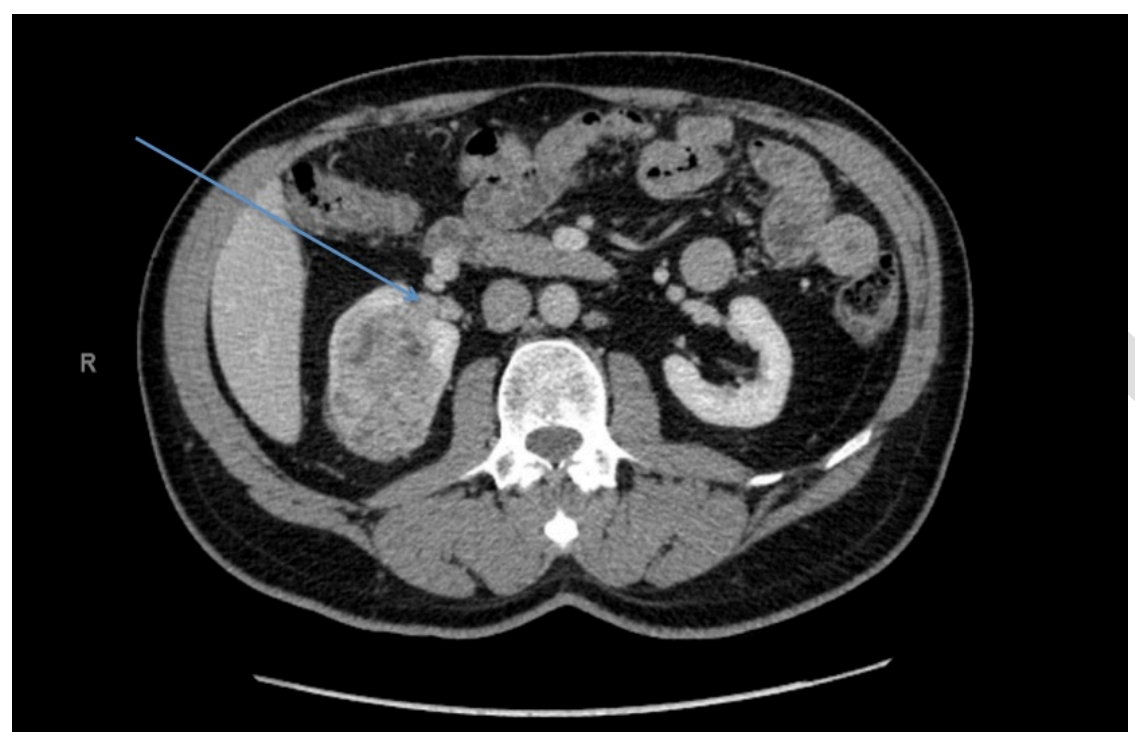

Fig. 2. Abdominal CT with IV contrast. Arrow indicating early venous infiltration into most cephalad right renal vein.

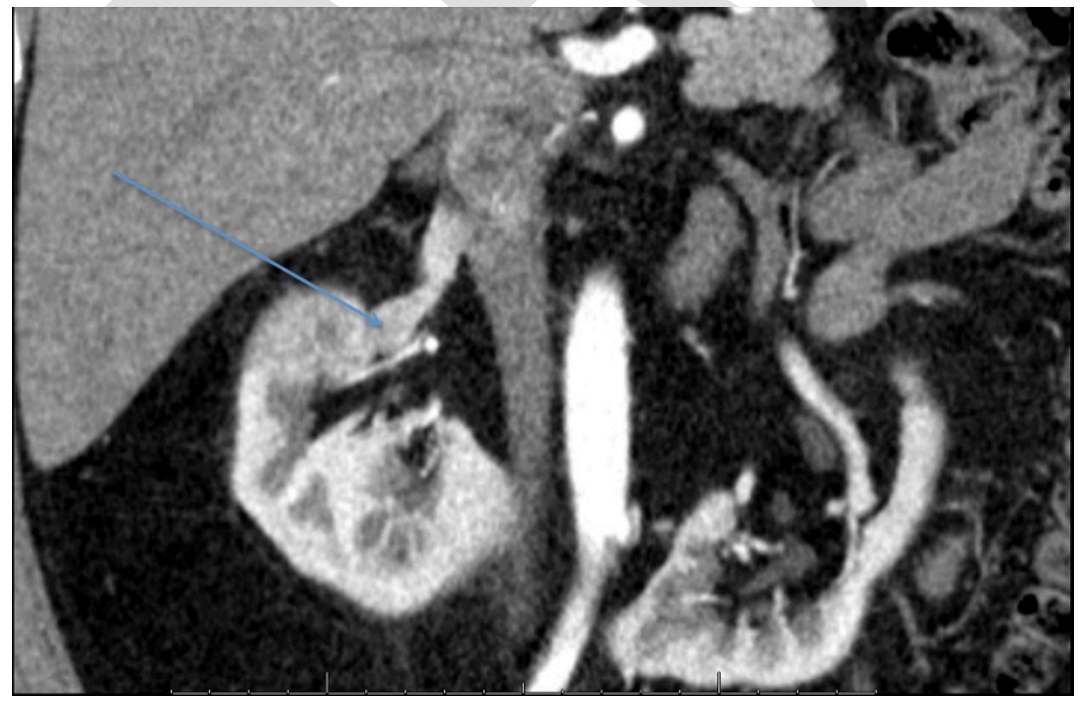


Fig. 3. Intra-operative images from laparoscopic procedure. (A) Most cephalad renal vein with tumor thrombus being transected with laparoscopic stapler. (B) Use of intra-operative ultrasound to confirm regional absence of perfusion with selective renal arterial clamping. (C) Closure of arteriotomy in segmental artery. (D) Renal resection bed with FloSeal; resected mass in background.

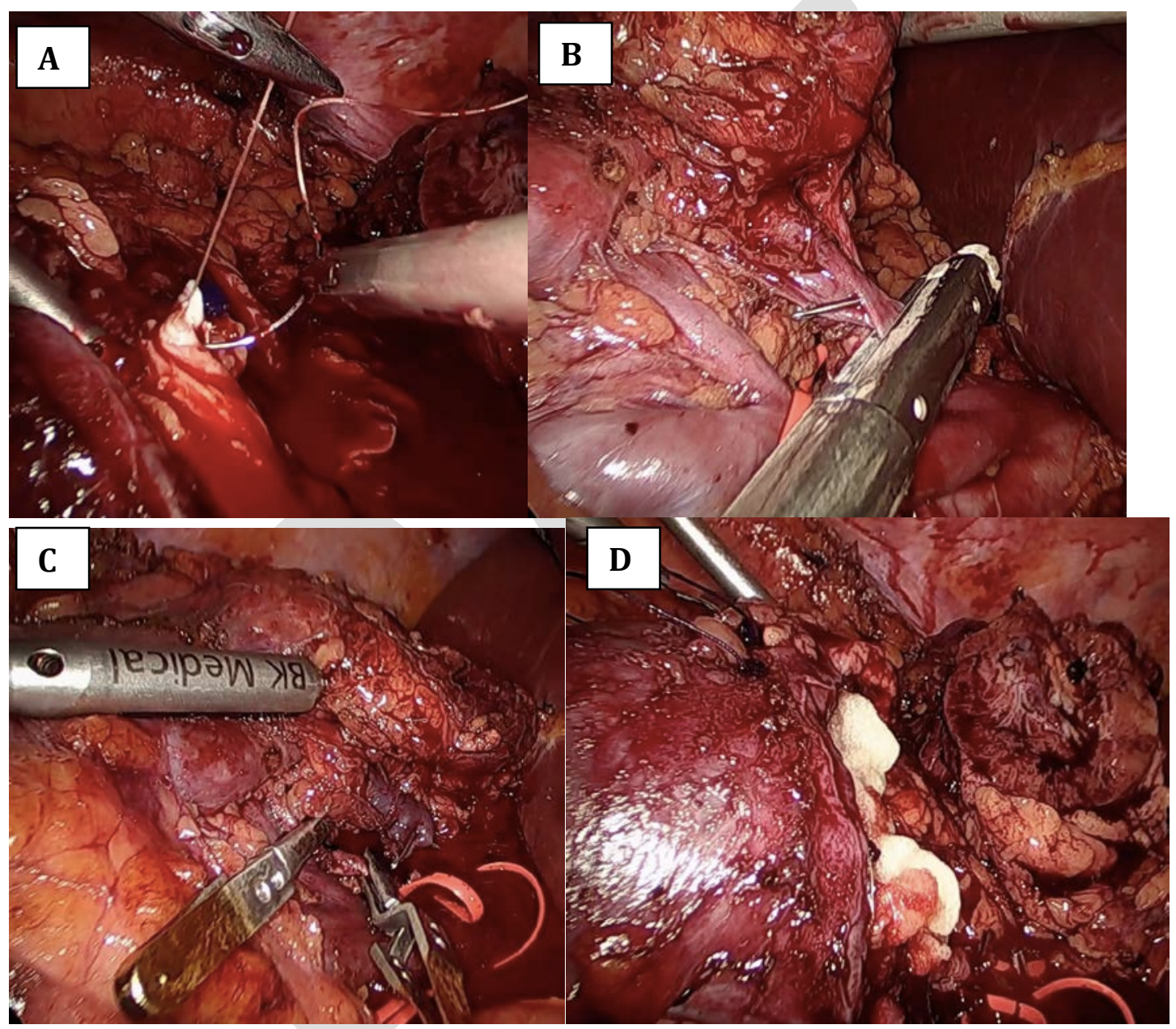

